\title{
Generalized s-topological Groups
}

\author{
Rehman Jehangir ${ }^{1, *}$, Moizud Din Khan ${ }^{2, *}$ \\ ${ }^{1}$ Department of Mathematics, Preston University Kohat (Islamabad), Pakistan \\ ${ }^{2}$ Department of Mathematics, COMSATS institute of information technology, Chak Shehzad Islamabad, Pakistan \\ *Corresponding author: Jehangir_pk@yahoo.com, moiz@comsats.edu.pk
}

Received July 12, 2015; Revised August 28, 2015; Accepted September 21, 2015

\begin{abstract}
In this paper, we explore the notion of generalized semi topological groups. This notion is based upon the two ideas, generalized topological spaces introduced by Csaszar [2,3] and the semi open sets introduced by Levine [7]. We investigate on the notion of generalized topological group introduced by Hussain [4]. We explore the idea of Hussain by considering the generalized semi continuity upon the two maps of binary relation and inverse function.
\end{abstract}

Keywords: generalized semi open sets, generalized compact sets, generalized continuity, generalized continuity, generalized discrete sets

Cite This Article: Rehman Jehangir, and Moizud Din Khan, "Generalized s-topological Groups.” Turkish Journal of Analysis and Number Theory, vol. 3, no. 4 (2015): 108-110. doi: 10.12691/tjant-3-4-4.

\section{Introduction}

Let $\mathcal{G}$ denotes the generalized topological space $(\mathrm{X}, \mathcal{G})$. In accordance with [3], let $\mathrm{A} \subseteq \mathrm{X}$ be generalized semi open if and only if there exists a generalized open set $(\mathcal{G}$-open set) $0 \in \mathcal{G}$ such that $0 \subseteq \mathrm{A} \subseteq \mathrm{cl}_{\mathcal{G}}(0)$, where $\operatorname{cl}_{\mathcal{G}}(0)$ denotes the generalized closure of the set $\mathrm{O}$ in $\mathcal{G}$. For more details on generalized topological spaces, we refer to [2,3]. In 2013, Murad et al. [4], defined and studied the concept of generalized topological groups ( $\mathcal{G}$-topological groups). This study was further extended and published in [5] and [6]. In 2015, C. Selvi and R. Selvi [10] were motivated by $\mathcal{G}$-topological groups [4] and S-topological groups [9], and defined on new notion with the name of generalized Stopological groups.

In this paper, we intend to generalized further the notion of $\mathcal{G}$-topological groups and $\mathcal{G}$-S-topological groups by using $\mathcal{G}$-semi continuity. $\mathcal{G}$-semi continuity is a generalization of $\mathcal{G}$-continuity and it was defined by Á. Császár in [3].

\section{Generalized Semi Topological Group}

In this section, we will explore the notion of generalized semi topological group. Generalized semi topological groups contains the structure of generalized topology and groups. The whole idea is backed by the generalized semi continuity, as the binary operation and the inverse map undergo the process of generalized semi continuity. We will study the basic definitions and gradual development of the phenomenon.

Lemma 2. 1. Let $\left(X, \mu_{1}\right)$ and $\left(Y, \mu_{2}\right)$ be generalized topological spaces and $\mathrm{f}: \mathrm{X} \rightarrow \mathrm{Y}$ is generalized semi continuous, then for any subset $\mathrm{A}$ of $\mathrm{X}, f(\operatorname{scl}(A) \subseteq$ $\operatorname{cl}(f(A))$.
Theorem 2. 2. Let $\left(\mathcal{T}_{1}, \mu_{1}\right)$ and $\left(\mathcal{T}_{2}, \mu_{2}\right)$ be generalized topological spaces and let $\left(\mathcal{T}_{1} \times \mathcal{T}_{2}, \mu_{1} \times \mu_{2}\right)=(\mathcal{T}, \mu)$ be their product generalized topological space if $A_{1}$ is generalized semi open set in $\mathcal{T}_{1}$ and $A_{2}$ is generalized semi open in $\mathcal{T}_{2}$, then $A_{1} \times A_{2}$ is generalized semi open in $(\mathcal{T}, \mu)$.

Proof. Assume that $A_{i}=O_{i} \cup B_{i}$, where $O_{i}$ is generalized open in $X_{i}$ and $B_{i} \subset c l_{i} O_{i}-O_{i}$, for $i=1,2$. This is nowhere dense set as well. Then,

$$
\begin{aligned}
& A_{1} \times A_{2}=\left(O_{1} \cup B_{1}\right) \times\left(O_{2} \cup B_{2}\right) \\
& =\left(O_{1} \times O_{2}\right) \cup\left(B_{1} \times O_{2}\right) \cup\left(O_{1} \times B_{2}\right) \cup\left(B_{1} \times B_{2}\right)
\end{aligned}
$$

But $\mathrm{O}_{1} \times \mathrm{O}_{2}$ is generalized open set in $\mathrm{X}_{1} \times \mathrm{X}_{2}$ and $\left(B_{1} \times O_{2}\right) \cup\left(O_{1} \times B_{2}\right) \cup\left(B_{1} \times B_{2}\right) \subset c l \mu_{1} \times \mu_{2}\left(O_{1} \times O_{2}\right)$ $=c l \mu_{1} \times \mu_{2}\left(O_{1} \times O_{2}\right)$

Hence, $\mathrm{A}_{1} \times \mathrm{A}_{2} \subseteq\left(O_{1} \times O_{2}\right) \cup \operatorname{cl} \mu_{1} \times \mu_{2}\left(O_{1} \times O_{2}\right)$ $=c l \mu_{1} \times \mu_{2}\left(O_{1} \times O_{2}\right)$

$$
O_{1} \times O_{2} \subseteq A_{1} \times A_{2} \subseteq c l \mu_{1} \times \mu_{2}\left(O_{1} \times O_{2}\right)
$$

This proves that $A_{1} \times A_{2}$ is generalized semi open set in $\mathrm{X}_{1} \times \mathrm{X}_{2}$.

Theorem 2. 3. Let: $(X, \mu) \rightarrow(Y, \sigma)$ semi generalized continuous map between two generalized topological spaces. Let $B$ be semi generalized compact set relative to $(X, \mu)$ then $f(B)$ is semi generalized compact in $(Y, \sigma)$.

Proof. Let $\left\{U_{i}: i \in \nabla\right\}$ be any collection of generalized open set of $(X, \mu)$, such that $f(B) \subset \cup\left\{U_{i}: i \in \nabla_{0}\right\}$. Then $B \subset \cup\left\{f^{-1}\left(U_{i}\right): i \in \nabla\right\}$ holds by hypothesis and there exists a finite subset of $\nabla_{0}$ of $\nabla$ such that $B \subset \cup\left\{f^{-1}\left(U_{i}\right)\right.$ : $\left.i \in \nabla_{0}\right\}$ which shows that $f(B)$ is semi generalized compact in $(Y, \sigma)$.

\section{Semi Generalized Topological Group}


In this section, we will define semi generalized topological groups ( $\mathcal{G}$-s-topological groups) and investigate its basic properties.

Definition 3. 1. $(X, *, G)$ is said to be a $\mathcal{G}$ - s-topological group if

(1). $(X, \mathcal{G})$ is generalized topological space;

(2). $(X, *)$ is a group;

(3). The multiplication map $m: X \times X \rightarrow X$, defined by $m(x, y)=x * y$ and the inverse map $i: X \rightarrow X$ defined by $i(x)=x^{-1} ; \forall x \in X$ are the generalized semi-continuous. Equivalently, $(X, *, \mathcal{G})$ is semi generalized topological group if $\forall x, y \in X$ and for each generalized open set $W$ containing $x * y^{-1} \in X$, there exist generalized semi open sets $U$ containing $x$ and $V$ containing $\mathrm{y}$, such that, $U * V^{-1} \in W$. Since every $\mathcal{G}$ - continuous is $\mathcal{G}-$ semi - continuous therefore, every $\mathcal{G}$-topological group is $\mathcal{G}-S$-topological groups. And $\mathcal{G}-S$-topological group may not be a $\mathcal{G}$-topological group. Further, we note that, every $\mathcal{G}$-topological group is $\mathcal{G}$-s-topological group and every $\mathcal{G}$ s-topological group is $\mathcal{G}$-S -topological group. However, converses may not be true in general. It is evident from the following example:

Example 3.2. Let $\Gamma=Z_{2}=\{0,1\}$ be the two-element (cyclic) group with the multiplication mapping $\mu=+2$ the usual addition modulo 2. Equip $\Gamma$ with the Sierpinski topology $\tau=\{\{\varphi\},\{0\}, \Gamma\}$. Then the collection of all the semi open sets

SO

$$
(\tau \times \tau)=\left\{\begin{array}{l}
\{(0,0)\},\{(0,0),(0,1)\}, \\
\{(0,0),(1,0)\},\{(0,0),(0,1),(1,0)\}, \\
\{(0,0),(0,1),(1,0),(1,1)\}, \\
\{(0,0),(1,1)\},\{(0,0),(0,1),(1,1)\}, \\
\{(0,0),(1,0),(1,1)\}
\end{array}\right\}
$$

and that $\mu: \tau \times \tau \rightarrow \tau$ is continuous at $(0,0)$, $(1,0),(0,1)$, but not continuous at $(1,1)$. However, $\mu$ is semi-continuous at $(1,1)$. For this, let us take the open set $\varsigma=\{0\}$ in $\Gamma$ containing $\mu(1,1)=0$. Then the semi-open set $\mathrm{Y}=\{(0,0),(1,1)\} \subset \tau \times \tau$ contains $(1,1)$. The inverse mapping $i: \tau \rightarrow \tau$ is continuous and hence semicontinuous. Therefore, $(\Gamma,+2, \tau)$ is a $\mathcal{G}-s$-topological group which is not a topological group. It was noticed in [1] that $(\Gamma,+2, \tau)$ is not a $\mathcal{G}-S$-topological group.

Theorem 3.3. Let $(X, *, \mu)$ be a generalized stopological group. Let $i:(X, \mu) \rightarrow(X, \mu)$ be an inverse mapping defined by $i(x)=x^{-1} ; \forall x \in X$. Then $i$ is generelized semi continuuous mapping.

Proof. Let $x \in X$. Let $\mathrm{W}$ be a generalized open set in $X$ containing $x^{-1}$. Then by hypothesis, there exist generalized semi open sets $\mathrm{U}$ and $\mathrm{V}$ containing $e$ and $x$, respectively, such that $U * V^{-1} \subseteq W$. In particular, $i(V)=V^{-1}=\mathrm{e} * V^{-1} \subseteq U * V^{-1} \subseteq W$.

Theorem 3.4. If $K$ is semi generalized compact, then $y * K^{-1}$ is semi generalized compact in a semi generalized topological group $(X, *, \mu)$.

Proof. Let $\left\{U_{\alpha}: \alpha \in \mathrm{I}\right\}$ be a cover of $y * K^{-1} \subset$ $\bigcup_{\alpha \in I} U_{\alpha}$. This implies that $K^{-1} \subset y^{-1} \star \bigcup_{\alpha \in I} U_{\alpha}=$ $\bigcup_{\alpha \in I} y^{-1} * U_{\alpha}$. This implies that $K \subset \bigcup_{\alpha \in I} y \star U_{\alpha}{ }^{-1}$.
Since $\mathrm{K}$ is semi generalized compact, then there exists a finite set $I_{0}$ of I such that $y^{-1} \star K \subset \bigcup_{\alpha \in I_{0}} U_{\alpha}^{-1}$. This implies that $\mathrm{y} * K^{-1} \subset \mathrm{U}_{\alpha \in \square_{0}} U_{\alpha}$. That is $y * K^{-1}$ has a finite subcover of $\mathrm{X}$. Hence $y * K^{-1}$ is semi generalized compact.

Theorem 3.5. A non empty subgroup $H$ of a semi generalized topological group is semi open if and only if its semi interior is non empty.

Proof. Assume that $x \in S_{\mu}-\operatorname{int}(H)$ (semi generalized interior). Then by definition there is a semi generalized open set $\mathrm{V}$ such that $x \in V \subset H$ : For every $y \in H$, we have $\mathrm{y} * V \subset y \subset H=H$. Since $\mathrm{V}$ is semi generalized open so is $y * V$, we conclude that $H=\cup\{y * V: y \in$ $H\}$ is a semi generalized open set as the union of semi generalized open sets is semi generalized open. Converse of this theorem is quite simple.

Lemma 3. 6. Let $\left(X, \mu_{1}\right)$ and $\left(Y, \mu_{2}\right)$ be generalized topological spaces and $f: X \rightarrow Y$ is generalized semi continuous, then for any subset $A$ of $X, f(\operatorname{scl}(A) \subseteq$ $\operatorname{cl}(f(A))$.

Further theorem is the extension of the work presented by Bohn Lee [1].

Theorem 3. 7. Let $(X, *, \mu)$ be a semi generalized topological group. Then for each generalized open set $X$ subset $A$ of $X ; A^{-1}$ is semi generalized open.

Proof. Let $A$ be generalized open in $X$, there exists a generalized open set $U$ in $X$, such that,

$$
\begin{gathered}
U \subseteq A \subseteq c l(U)(\operatorname{By}[5]) \\
\Rightarrow U^{-1} \subseteq A^{-1} \subseteq[\operatorname{cl}(\mathrm{U})]^{-1}
\end{gathered}
$$

Because, $(X, *, \mu)$ is semi generalized topological group.

Lemma 3. 8. Let $A$ be $\mathcal{G}$-topological space. If $A \subseteq X$ is $\mathcal{G}$ semi open and $A \subseteq B \subseteq \operatorname{cl}_{\mathcal{G}}(A)$, then $B$ is $\mathcal{G}$-semi open.

Proof: Let $A \subseteq B \subseteq c l_{\mathcal{G}}(A)$. Since $A$ is semi $\mathcal{G}$-open therefore there exists a $\mathcal{G}$-open set $O$ such that $O \subseteq A \subseteq$ $c l_{\mathcal{G}}(O)$.

$\Rightarrow O \subseteq A \subseteq B$ implies that $O \subseteq B \subseteq \operatorname{cl}_{\mathcal{G}}(O)$.

This proves that $B$ is semi $\mathcal{G}$-open in $X$.

Theorem 3.9. Let $(X, *, \mathcal{G})$ be a $\mathcal{G}$-s-topological group. Then the multiplication mapping

$$
M: \mathcal{G} \times \mathcal{G} \rightarrow \mathcal{G} \text { defined by } m((x, y))=x * y
$$

is semi $\mathcal{G}$-continuous for each $x, y \in X$.

Proof: Let $x, y \in X$ and $\mathrm{W}$ be a $\mathcal{G}$-open set containing $x * y$, since $\mathrm{X}$ is $\mathcal{G}$-semi open sets $U$ and $V$ containing $x$ and $y^{-1}$, such that

$$
\begin{aligned}
& U^{*} V^{-1} \subset W \\
\Rightarrow & x^{*} y \in U^{*} V^{-1} \subset W \\
\Rightarrow & m((x, y))=x^{*} y \in U^{*} V^{-1} \subset W \\
\Rightarrow & m\left(U \times V^{-1}\right)=U^{*} V^{-1} \subset W
\end{aligned}
$$

Since $V$ is semi $\mathcal{G}$-open set containing $y^{-1}$, therefore, by Theorem-1.6, $V^{-1}$ is $\mathcal{G}$-semi open set containing y. Moreover, by Theorem $2, U \times V^{-1}$ is $\mathcal{G}$-semi open set containing $(x, y)$. Hence ' $m$ ' is semi $\mathcal{G}$-continuous for each $(x, y)$.

By Theorems 2.5 and 2.7, it is clear that every $\mathcal{G}$-stopological group is $\mathcal{G}$-S -topological group. 
Theorem 3.10. Let $(X, *, \mathcal{G})$ be a $\mathcal{G}$-s-topological group and $A$ be any $\mathcal{G}$-open set in $X$. Then, for each $x \in X, x * A$ both are $\mathcal{G}$-semi open in $X$.

Proof: Let $z \in A * x$. This gives $z=y * x$ for some $y \in A$.

$$
\Rightarrow y=z^{*} x^{-1} \in(A * x) * x^{-1} .
$$

Since, $X$ is $\mathcal{G}$-s-topological group, therefore, for $\mathcal{G}$-open set A containing $y$, there exist $\mathcal{G}$-open set $U$ and $V$ containing $z$ and $x$ respectively, such that

$$
U^{*} V^{-1} \subseteq A .
$$

Or

$$
z^{*} x^{-1} \in U^{*} x^{-1} \in U^{*} V^{-1} \subset A .
$$

This gives $z \in \subset A * x$. This proves that $A * x$ is $\mathcal{G}$ semi open set.

Theorem 3.12. Let $(X, *, \mu)$. be semi $\mathcal{G}$-topological group. If $A$ is generalized open and $B \subseteq X$, then $A *$ $B, B * A$ is generalized semi open in $X$.

Proof. Let $x \in B$ and $z=A * x$

$$
z=y * x
$$

or, for some $\psi \in \mathrm{A}=(\mathrm{A} * \xi) * x^{-1}$

Now, $z, x \in X$, implies,

$$
\mathrm{z} * x^{-1} \in A
$$

Where $A$ is generalized open set in $X$, therefore, by the hypothesis, i.e., $X$ is semi generalized topological group, there exist generalized semi open set in $\mathrm{X}$ containing $\mathrm{z}$ and $V$ containing $x$ such that,

$$
U^{*} \mathrm{~V}^{-1} \subseteq A
$$

or

$$
U^{*} x^{-1} \subseteq U^{*} \mathrm{~V}^{-1} \subseteq A
$$

or

$$
U \subseteq A^{*} x .
$$

This implies that for each point $\mathrm{z} \in A * \mathrm{x}$, we can find a generalized semi open set $\mathrm{U}$ containing z such that $U \subseteq A$ $* \mathrm{x}$. This means $A * \mathrm{x}$ is generalized semi open. Since the union of semi open sets is generalized semi open, therefore,

$$
A^{*} B=\bigcup_{x \in B} A^{*} \mathrm{x}
$$

is generalized semi open.

Lemma 3. 13. Let $(X, *, \mathcal{G})$ be a semi $\mathcal{G}$-topological group and let $\mathcal{B}$ be the base at identity element e of $X$. Then, for every $U \in \mathcal{B}$, there is an element $V \in S O(X)$; so that following holds,

1) $V^{2} \subset \mathrm{U}$

2) $V^{-1} \subset \mathrm{U}$.

3) $\quad V * x \subset U$, for each $x \in U$.

Theorem 3.14. Let $(X, *, \mathcal{G})$ be a semi $\mathcal{G}$-topological group. Then each left(right) translation $l_{g}: \mathcal{G} \rightarrow$ $\mathcal{G}\left(r_{g}: \mathcal{G} \rightarrow \mathcal{G}\right)$ is $\mathcal{G}$-semi homeomorphic.

Proof: It is obviously bijective map and $l_{g}: \mathcal{G} \rightarrow \mathcal{G}$ is semi $\mathcal{G}$-continuous containing $g x$, there exists $\mathcal{G}$-semi open set $U$ containing x such that $l_{g}(U) \subseteq W$. Again, let $V$ be a $\mathcal{G}$-open set in $\mathcal{G}$, then $l_{g}(V)=V * g$ is semi $\mathcal{G}$-open. That is the image of $\mathcal{G}$-open set is semi $\mathcal{G}$-open. This proves that $l_{g}$ is $\mathcal{G}$-semi homeomorphic.

Note: Let $(X, *, \mathcal{G})$ be semi $\mathcal{G}$-topological group and $x \in X$. Then for any local base $\beta_{e}$ and $\mathrm{e} \in \mathcal{G}$, then each of the families $\beta_{x}=\left\{x * U: U \in \beta_{e}\right\}$ and $\left\{x * U^{-1}: U \in \beta_{e}\right\}$ is a semi $\mathcal{G}$-open neighborhood system of $x$.

\section{References}

[1] E. Bohn, J. Lee, Semi-topological groups, Amer. Math. Monthly 72 (1965), 996.998.

[2] Á. Császár, Generalized open sets, Acta Math. Hungar., 75 (1997), 65-87.

[3] Á. Császár, Generalized topology, generalized continuity, Acta Math.Hungar., 96 (2002), 351-357.

[4] M. Hussain, M. Khan and C. Ozel, On generalized topological groups, Filomat, 2013 27(4):567-575.

[5] M. Hussain, M. Khan and C. Ozel, More on generalized topological groups, Creative Math. \& Inf. 22(2013)(1), 47-51.

[6] M. Hussain, M. Khan, A.Z. Özcelik and C. Ozel, Extension closed properties on generalized topological groups, Arab J Math (2014) 3:341-347.

[7] N. Levine, Semi-open sets and semi-continuity in topological spaces, Amer. Math. Monthly 70 (1963), 36.41.1.

[8] Michel Coste, Real Algebraic Sets, March 23, 2005.

[9] J. Cao, R. Drozdowski, Z. Piotrowski, Weak continuity properties of topologized groups, Czech. Math. J., 60 (2010), 133-148.

[10] C. Silva and R. Silva, On generalized S topological group, International journal of science and research, 6, (2015), 1-4. 